\title{
Do Self-Reported Traits and Aggregated States Capture the Same Thing? A Nomological Perspective on Trait-State Homomorphy
}

Social Psychological and Personality Science 2019, Vol. 10(5) 596-6II

(C) The Author(s) 2018

Article reuse guidelines:

sagepub.com/journals-permissions DOI: 10.1 | $77 / 19485506 / 8774772$ journals.sagepub.com/home/spp

(QSAGE

\author{
John F. Rauthmann', Kai T. Horstmann², and Ryne A. Sherman ${ }^{3,4}$
}

\begin{abstract}
Convergent correlations between traits and state aggregates from experience sampling cannot fully establish trait-state homomorphy (the extent to which the same constructs are measured). With a nomological vector correlation and lens model approach, we test how similar nomological networks of traits and state aggregates are to each other: A trait and state-aggregate capture the same construct when both show highly similar nomological associations to a common set of correlates. In large experience sampling $(N=209)$ and life-logging studies $(N=298)$, Extraversion, Conscientiousness, and Agreeableness tended to show more and Openness, Honesty/Humility, and Neuroticism/Emotionality tended to show less trait-state homomorphy. However, these general findings differed somewhat at the aspect level, with Neuroticism and Extraversion aspects tending to show more versus Openness and Honesty/Humility aspects tending to show less homomorphy. The proposed nomological approaches can be flexibly applied to other traits, states, and correlates.
\end{abstract}

\section{Keywords}

nomological network, lens model, trait, state, HEXACO, Big Five, DIAMONDS

In experience sampling studies, personality states (e.g., behaving extraverted at a given moment) - momentary manifestations or expressions of personality traits-are repeatedly assessed. In principle, the mean aggregate of these states across all measurements - the regularity in one's thoughts, feelings, desires, and behaviors - should approximate a trait (e.g., being generally an extraverted person). Indeed, research has shown that the mean of a density distribution of states (a state aggregate) is associated with self-reported trait measures $(r \mathrm{~s} \sim .20-.60$; Augustine \& Larsen, 2012; Finnigan \& Vazire, 2017; Fleeson, 2001; Fleeson \& Gallagher, 2009; Heller, Komar, \& Lee, 2007; Horstmann \& Rauthmann, in preparation). However, do assessments of traits and state aggregates really capture the same constructs? To answer this, we employ a nomological vector correlation (NoVeCA) and lens model approach (NoLeMA) to examine nomological trait-state homomorphy.

\section{Background}

Traits and States

Personality traits are conceptualized as stable interindividual differences in thoughts, feelings, desires, and behaviors (Funder, 2001). However, people also regularly exhibit a range of momentary states across situations (Fleeson, 2001, 2007;
Rauthmann, Jones, \& Sherman, 2016; Sherman, Rauthmann, Brown, Serfass, \& Jones, 2015) defined as "having the same affective, behavioral, and cognitive content as a corresponding trait (...), but as applying for a shorter duration" (Fleeson \& Jayawickreme, 2015, p. 84). Per Whole Trait Theory (Fleeson, 2012), states are momentary instantiations of traits and form distributions within persons. Thus, averaged state aggregates should approximate traits if trait and state measures were chosen diligently and enough situations were sampled representatively within participants' daily lives.

However, a crucial question is to what extent traits and state aggregates are homomorphous (Fleeson, 2001). Psychometrically, demonstrating substantial correlations between traits and state aggregates represents convergent validity. As construct validation is an open, ongoing, and iterative process (Campbell

\footnotetext{
' Wake Forest University, Winston-Salem, NC, USA

${ }^{2}$ Humboldt-Universität zu Berlin, Berlin, Germany

${ }^{3}$ Texas Tech University, Lubbock, TX, USA

${ }^{4}$ Hogan Assessment Systems
}

Corresponding Author:

John F. Rauthmann, Wake Forest University, 4I 5 Greene Hall, Winston-Salem, NC 27109, USA.

Email: jfrauthmann@gmail.com 
\& Fiske, 1959; Cronbach \& Meehl, 1955; Loevinger, 1957), it is prudent to examine also other forms of validity. Thus, we propose an additional focus on nomological validity - the extent to which two constructs, or their measures, show similar associations with a set of correlates (Cronbach \& Meehl, 1955; Hough, Oswald, \& Ock, 2015; Rauthmann \& Sherman, 2016a, 2016b).

\section{Nomological Networks}

Cronbach and Meehl (1955, p. 290) argued that "scientifically speaking, to 'make clear what something is' means to set forth the laws in which it occurs [and] (...) the interlocking system of laws which constitute a theory [shall be referred to] as a nomological network." This means that we can learn something about a construct by examining how it is associated with a set of other variables. It follows that we can define and compare scales in terms of their nomological networks. If two scales share a highly similar nomological network (nomological homomorphy), then we may pragmatically conclude that they both tap a highly similar construct — even if they are not highly correlated with each other.

This principle has already been used in developmental research, where it is important to establish that scales administered in early ages capture the same constructs as those administered in later ages. For example, Big Five scales in children show similar nomological networks to those of Big Five scales in adults (e.g., Asendorpf \& van Aken, 2003; Measelle, John, Ablow, Cowan, \& Cowan, 2005), with the conclusion that "the same" Big Five are measured across time-despite mean-level and rank-order differences (Roberts \& DelVecchio, 2000; Roberts, Walton, \& Viechtbauer, 2006). ${ }^{1}$ Further, in organizational research, Hough et al. (2015) highlighted how nomological web clustering can identify constructs with similar correlates so as to taxonomize them. Together, we may utilize a nomological perspective to understand to what extent traits and state aggregates are "the same thing" (trait-state homomorphy).

A nomological perspective. Nomological homomorphy can be examined with vector correlations or lens models. NoVeCA may represent the usual or traditional approach, although it has not been used to examine trait-state homomorphy yet. ANoLeMA is novel and has, to our knowledge, not yet been used to examine any questions at all.

NoVeCA. To examine their nomological similarity, traits and state aggregates can be correlated each separately with a set of correlates, and then the resulting two profiles of $r$-to-z transformed correlation coefficients are correlated. The resulting vector correlation indexes how similar nomological correlations were between traits and state aggregates. This piecemeal procedure is straightforward, easy to implement, and has already been utilized in literature (e.g., Miller et al., 2017; Rauthmann \& Sherman, 2016a, 2016b). It works especially well when many correlates are used. However, a drawback is that the NoVeCA does not consider intercorrelations among correlates. The NoLeMA addresses this issue by adopting multiple regressions instead of bivariate correlations.

NoLeMA. When nomological correlates are regressed on both the trait and the state aggregate, shared variance among correlates is considered. Such modeling is consistent with the mathematical formulation of a Brunswikian lens model (Brunswik, 1934, 1943, 1952; Hammond, 1980; Hogarth \& Karelaia, 2007; Karelaia \& Hogarth, 2008). Lens models are commonly used in personality/social psychology to study interpersonal perception (Nestler \& Back, 2013). Briefly, a trait is associated with several observable cues that may be used for trait judgments. The correlation between trait scores and judgments is termed achievement. Correlations between traits and cues denote cue validity and those between cues and judgments denote cue utilization. Here, we modify this terminology and say, correlations between traits and state aggregates denote trait-state manifestations, those between traits and correlates denote trait saturation, and those between correlates and state aggregates denote state saturation.

A schematic lens model (Hogarth \& Kareleia, 2007) is presented in Figure 1. The left side concerns trait saturation $R_{\text {trait }}$ which is high when trait data are strongly predictable from nomological correlates. It is modeled within a multiple regression framework as:

$$
\begin{aligned}
Y_{\text {trait }} & =\sum_{i=1}^{n} b_{\text {trait }_{i}} \cdot X_{i}+\varepsilon_{\text {trait }} \\
R_{\text {trait }} & =\rho_{Y_{\text {trait }} \hat{Y}_{\text {trait }}}
\end{aligned}
$$

where $Y_{\text {trait }}=$ trait data to be predicted, $b_{\text {trait }}=$ regression weights, $X_{i}, i=1, \ldots, n=$ nomological correlates 1 to $n$, and $\varepsilon_{\text {trait }}=$ residual error term. Such a regression yields a multiple $R$ that is the correlation between $Y_{\text {trait }}$ (actual values) and $\hat{Y}_{\text {trait }}$ (predicted values).

The right side of Figure 1 concerns state saturation $R_{\text {state, }}$, which is high when state data are strongly predictable from correlates. It is modeled as:

$$
\begin{aligned}
& Y_{\text {state }}=\sum_{i=1}^{n} b_{\text {state }_{i}} \cdot X_{i}+\varepsilon_{\text {state }} \\
& R_{\text {state }}=\rho_{Y_{\text {state }} \hat{Y}_{\text {state }}}
\end{aligned}
$$

where $Y_{\text {state }}=$ state data to be predicted, $b_{\text {state }}=$ regression weights, $X_{i}, i=1, \ldots, n=$ nomological correlates 1 to $n$, and $\varepsilon_{\text {trait }}=$ residual error term. Such a regression yields a multiple $R$ that is the correlation between $Y_{\text {state }}$ (actual values) and $\hat{Y}_{\text {state }}$ (predicted values).

Correlating the actual scores $Y_{\text {trait }}$ and $Y_{\text {state }}$ yields a traitstate manifestation index $r_{\text {trait.state }}$ (achievement $r_{\mathrm{a}}$ in lens model literature):

$$
r_{\text {trait.state }}=\rho_{Y_{\text {trait }} Y_{\text {state }}}
$$




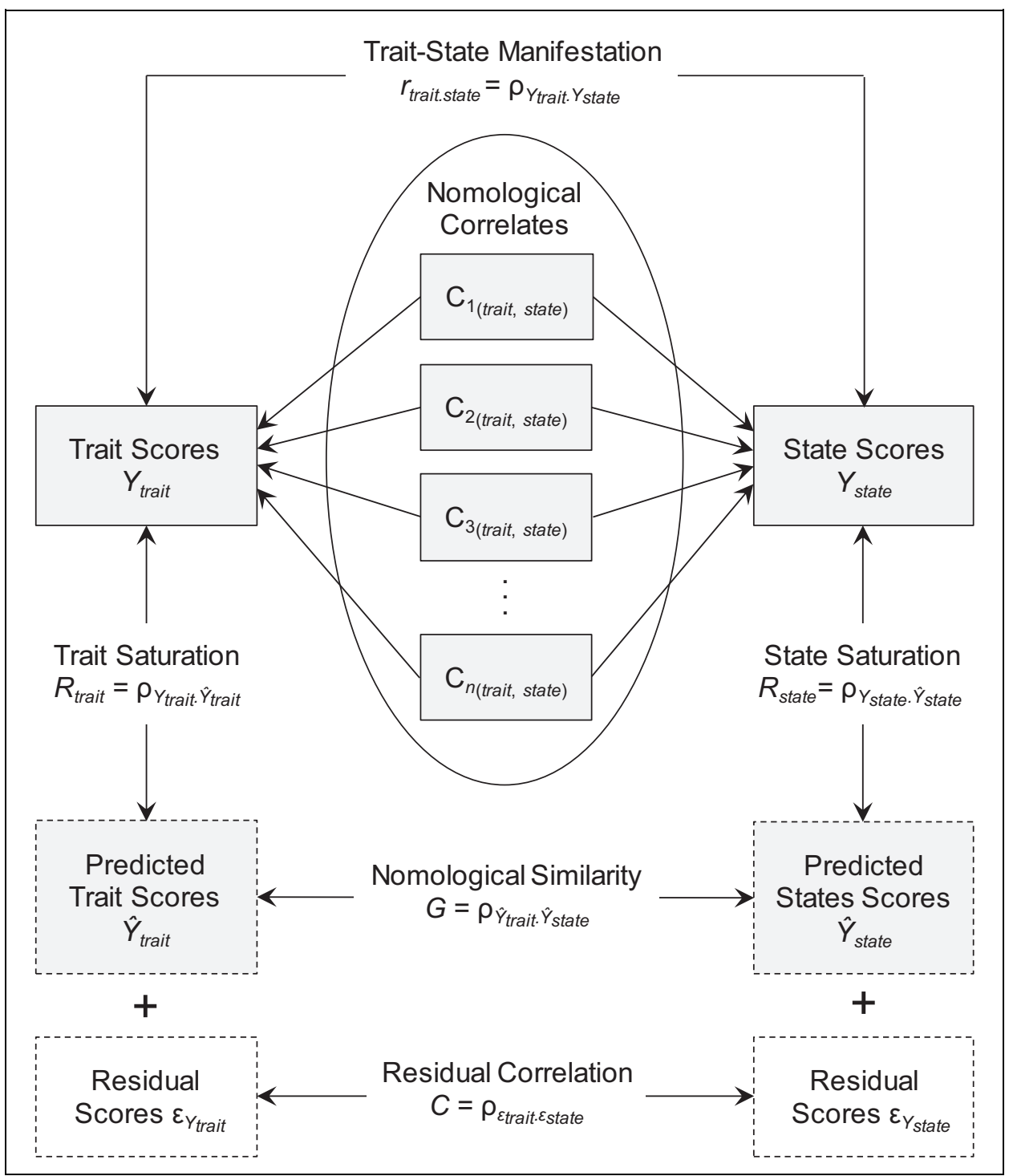

Figure I. Representation of a nomological lens model.

The convergent correlation between a trait and state aggregate can be diminished for several reasons (e.g., lack of reliability, not enough states aggregated, different measures, or items used). Thus, a small correlation alone is not necessarily indicative of a lack of trait-state homomorphy. Moreover, $r_{\text {trait.state }}$ is itself a function of other lens model indices as explained in Equation 6.

The predicted scores $\hat{Y}_{\text {trait }}$ and $\hat{Y}_{\text {state }}$ can be correlated to obtain a nomological similarity index, referred to as the matching index $G$ in lens model literature:

$$
G=\rho_{\hat{Y}_{\text {trait }} \hat{Y}_{\text {state }}}
$$

$G$ represents how well the linear trait saturation model matches that of state saturation: The more the weights and function forms of trait and state saturation correspond, the stronger the correspondence between traits and state aggregates. Put differently, the more similarly traits and state aggregates are associated with a set of common correlates, the higher $G$-and thus nomological homomorphy-will be. However, $G$ may be large even if weights do not correspond if there are high intercorrelations among correlates (Castellan, 1992; Dawes \& Corrigan, 1974; Einhorn \& Hogarth, 1975). ${ }^{2}$

The error scores $\varepsilon_{\text {trait }}$ and $\varepsilon_{\text {state }}$ can be correlated to obtain a residual correlation index, referred to as $C$ in the lens model literature:

$$
C=\rho_{\varepsilon_{\text {trait }} \varepsilon_{\text {state }}}
$$

$C$ captures the part of $r_{\text {trait.state }}$ that is not linearly accounted for due to (a) omitted correlates (i.e., not all might have been available/sampled), (b) configurality (i.e., interactions among correlates), (c) nonlinear relations between correlates and traits or state aggregates, or (d) some combination of these reasons (Gorman, Clover, \& Doherty, 1978). $C$ is 
equal to the partial correlation between $Y_{\text {trait }}$ and $Y_{\text {state }}$ controlled for all $n$ correlates $X_{i}$.

The indices $r_{\text {trait.state }}, G$, and $C$ are related such that $r_{\text {trait.state }}$ can be expressed within following lens model equation (Tucker, 1964, p. 528):

$$
r_{\text {trait.state }}=G \cdot R_{\text {trait }} \cdot R_{\text {state }}+C \cdot \sqrt{\left(1-R_{\text {tratt }}^{2} \cdot\left(1-R_{\text {state }}^{2}\right.\right.}
$$

This relation alerts us that the simple correlation $r_{\text {trait.state }}$ is composed of different aspects. Indeed, if $C$ was low or zero, Equation 6 would simplify to $r_{\text {trait.state }}$ being the product of $R_{\text {trait }}, R_{\text {state }}$, and $G$. Thus, we suggest computing all lens model indices to obtain a fuller picture of the commonly computed $r_{\text {trait.state }}$.

\section{The Current Work}

Trait-state manifestation concerns the extent to which a trait is expressed in an aggregate of states sampled across a limited range of situations and time. ${ }^{3}$ In statistical terms, we examine to what extent people's rank orders on a trait are preserved on a state aggregate. For various reasons, we may not expect a perfect rank ordering, and previous literature shows modest trait-state manifestations (Horstmann \& Rauthmann, in preparation). However, the extent to which the trait and state aggregate capture the same construct in their respective measurements is a different, though related, question. To address this question of trait-state homomorphy, we contend that the similarity of the nomological networks of a trait and a state aggregate is revealing. If a state aggregate shows a highly similar nomological network as the trait does, then both are relatively nomologically homomorphous.

We examined trait-state homomorphy in two studies with different sets of domains, aspects, and correlates. As traits and states of interest, we used two frameworks containing the widely used Big Five plus additional dimensions: HEXACO (Ashton \& Lee, 2007) in Study 1 and Big Five Aspects (BFAS: DeYoung, Quilty, \& Peterson, 2007) in Study 2. As nomological correlates, we selected a broad range of person and situation characteristics in both studies.

For person characteristics correlates, we used a measure that assesses a broad variety of enduring traits. Toward this end, we deemed the California Adult Q-Sort (CAQ; Bem \& Funder, 1978; Block, 1961, 2008) ideal with its 100 diverse person descriptors, devised to comprehensively measure important person characteristics. Using the CAQ items as correlates is especially fruitful in the NoVeCA that works best with many correlates.

For situation characteristics correlates, we considered theory and research linking persons, situations, and behaviors (Funder, 2006, 2008). Because states are manifested in situations (Fleeson \& Jayawickreme, 2015), it makes sense to use situation characteristics as important correlates. We used the Situational Eight DIAMONDS taxonomy that (a) affords a reasonably comprehensive assessment of situation characteristics (Rauthmann et al., 2014; Rauthmann \& Sherman, 2016a, 2016b), (b) is conceptually linked to person variables (Rauthmann, 2016), and (c) shows replicable and meaningful empirical relations to traits and states (Horstmann \& Ziegler, in press; Rauthmann, Sherman, Nave, \& Funder, 2015; Rauthmann et al., 2014, 2016). Using the DIAMONDS as correlates is especially fruitful in the NoLeMA that works best with few predictors.

\section{Method}

\section{Participants and Procedures}

Study I. These data have already been used by Sherman et al. (2015), Rauthmann et al. (2016), and Jones, Brown, Serfass, and Sherman (2017). From 218 ethnically diverse undergraduates at the Florida Atlantic University participating for partial course credit, data from 209 were usable for the current analyses $(67.94 \%$ women; age: $M=18.64, S D=1.86)$. In the first part of the study, participants provided informed consent, were interviewed, and completed different measures. From this part, we used personality traits and life situations. In the second part, participants received a text message 8 times per day ( 9 a.m. -11 p.m.) for seven consecutive days, prompting them to fill out an online survey on their current states and characteristics of the situation they were in. On average, participants completed about 39 reports. More information on exact experience sampling procedures and preprocessing of data can be found in Sherman et al. (2015, pp.877-878).

Study 2. Of 298 participants, the majority were undergraduate students $(58.72 \%$ women, $0.67 \%$ no indication; age: $M=21.11, S D=6.21)$. In the first part of the study, participants provided informed consent, were interviewed, and completed a variety of personality measures. From this part, we used personality traits. In the second part, participants wore a small photographic camera on their outermost layer of clothing for the duration of their next waking day. The camera automatically took a photograph at 30-s intervals while worn. The following day, participants returned to the laboratory with the camera and were shown their photos. After first being allowed to privately delete any photos they did not want to share, participants divided their photos into situation segments, effectively marking where one situation ended and another began. Participants then rated situation characteristics and states in each situation segment. More information can be found in Brown, Blake, and Sherman (2017).

\section{Measures}

Table 1 summarizes all measures for traits, states, and correlates in Studies 1 and 2. Study 1 used HEXACO scales and Study 2 Big Face Aspect Scales plus Honesty and Humility as additional aspects. Further, both studies used as nomological correlates CAQ items (measuring enduring person characteristics) and aggregated daily situations, while Study 1 additionally used life situations (i.e., enduring characteristics of one's typical situations in current life). It is desirable to use nomological correlates that are gathered before, during, or after experience 


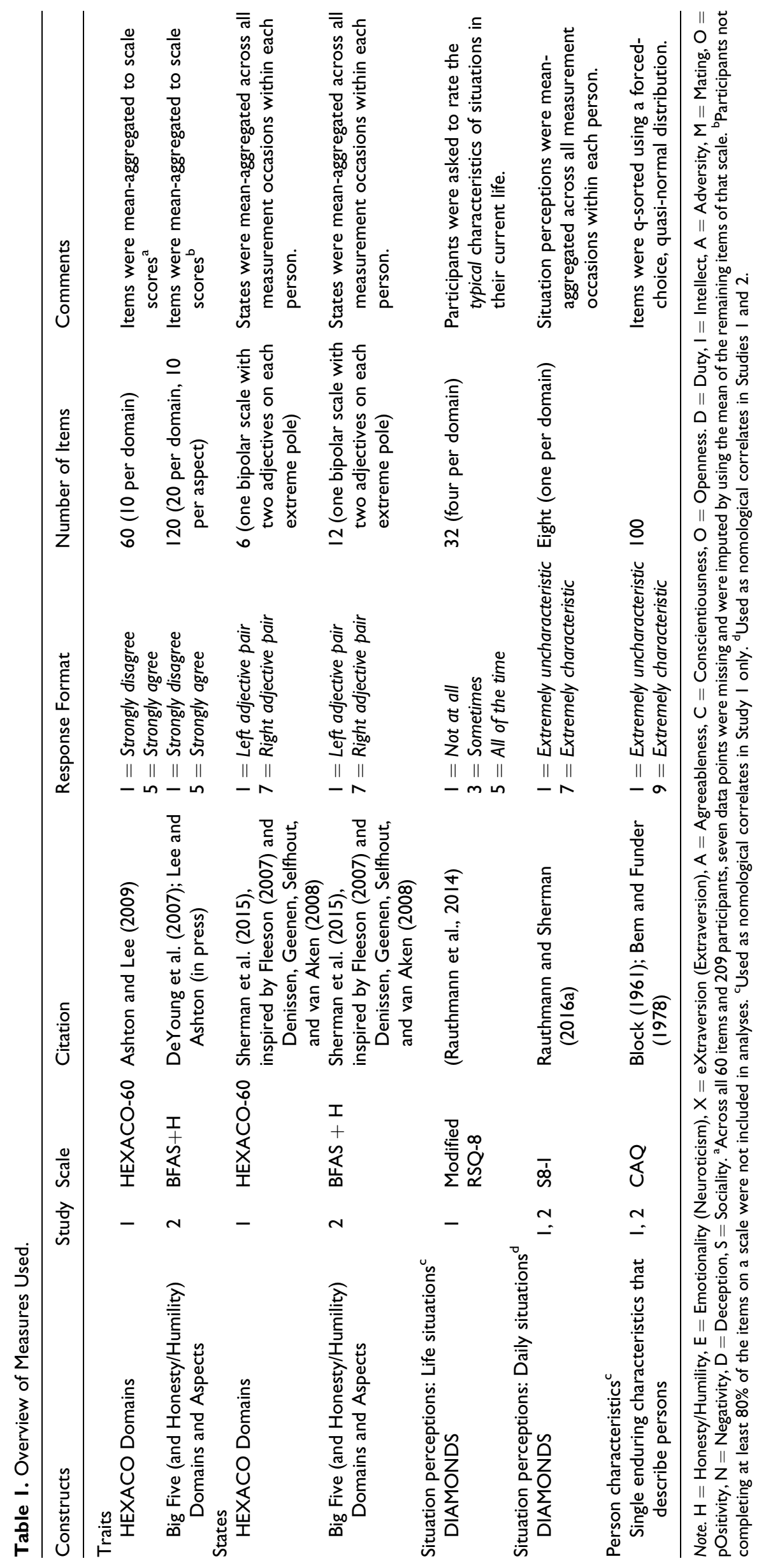


sampling. Thus, we considered both aggregated daily situation characteristics (assessed during experience sampling along with behavioral states) and midterm stable life situation characteristics and CAQ person characteristics (assessed before experience sampling along with the traits).

\section{Data Analysis}

All data and reproducible R code (also customizable to other data sets) can be found openly accessible at https://osf.io/ $3 \times 9 r 5$. Data were analyzed in R (R Core Team, 2016) and RStudio (RStudio Team, 2015) with the following packages: multicon (Sherman, 2015; Sherman \& Serfass, 2015), psych (Revelle, 2014), purrr (Wickham, 2016), broom (Robinson, 2016), lme4 (Bates, Maechler, Bolker, \& Walker, 2014), xlsx (Dragulescu, 2014), and knitr (Xie, 2016). We performed lens model analyses for three sets of nomological correlates: eight aggregated daily situation characteristics (Studies 1 and 2), eight life situation characteristics (Study 1), and $100 \mathrm{CAQ}$ items (Studies 1 and 2). Because we were interested in nomological homomorphies between traits and state aggregates, we mean aggregated daily situation characteristics within participants.

For a NoVeCA, we first correlated traits and state aggregates each with all nomological correlates. This resulted in vectors of correlations, which were $r$-to- $z$ transformed and then correlated with each other. For a NoLeMA, we performed lens model analyses using the lensModel function from the multicon package, computing trait, and state saturations as well as $r, G$, and $C$ indices. The NoVeCA approach works best with many correlates (i.e., $100 \mathrm{CAQ}$ items) as a smaller number of correlates (e.g., Eight DIAMONDS) means a lower reliability of the resulting vector correlations (Sherman \& Wood, 2014). In contrast, the NoLeMA works best with fewer predictors (i.e., Eight DIAMONDS) as too many predictors (e.g., 100 CAQ items) may introduce problems of power (larger sample sizes are needed for more predictors), multicollinearity, suppression, and misspecification. These caveats should be taken into account when interpreting findings.

\section{Results}

Descriptive statistics of all variables can be found in Table 2 . Table 3 presents, at the domain and aspect level, trait-state manifestations and a summary of all findings from the NoVeCA (Online Supplemental Tables A, B, and C) and NoLeMA (Online Supplemental Tables A, D, and E) for Studies 1 and 2. Figure 2 contains a graphical overview of focal findings. Readers interested in the details are referred to the supplemental tables and https://osf.io/3x9r5.

\section{Trait-State Manifestations}

As seen in Table 3 under $r$, most trait-state manifestations (correlations between measures of traits and state aggregates) were small to moderate in both studies for domains (average $r=.27$, range: -.03 for Study 2 Honesty/Humility to .41 for Study 2 Extraversion) and aspects (average $r=.26$, range: .03-.43). As explained previously, this is only one part of a larger picture; indeed, the indices $r, G$, and $r_{v}$ were, on average, substantially correlated when correlating the $r$-to- $z$ transformed vectors of coefficients from Table $3(r \mathrm{~s}=.73-.81$, $p$ s $<.001)$. Nomological analyses, however, reveal more about to what extent traits and state aggregates were homomorphous.

\section{Nomological Vector Correlations}

Domains. Domain-level correlations of traits and state aggregates, respectively, with nomological correlates (DIAMONDS, CAQ) can be found in Online Supplemental Table B for both studies. These correlations served, after $r$-to- $z$ transformation, as input for vector correlations, found in Table 3 under $r_{v}$. Most $r_{v}$ S were substantial (average $=.84$ ) and statistically significant. Of the $30 r_{v}$ coefficients, six had $p$ values $>.05$ (see light-gray coefficients); these pertained to Honesty/Humility, Emotionality, and Openness. In contrast, eXtraversion, Agreeableness, and Conscientiousness showed the highest $r_{v} \mathrm{~s}$ and thus higher nomological trait-state homomorphy.

Aspects. Aspect-level correlations of traits and state aggregates, respectively, with nomological correlates (DIAMONDS, CAQ) can be found in Online Supplemental Table $\mathrm{C}$ for both studies. These correlations served, after $r$-to- $z$ transformation, as input for vector correlations, found in Table 3 under " $r_{v}$." Most $r_{v}$ s were substantial (average $=.81)$ and statistically significant. Of the $24 r_{v}$ coefficients, two had $p$ values $>.05$ (see light-gray coefficients); these pertained to Honesty and Intellect. In contrast, Enthusiasm, Withdrawal, Volatility, and Assertiveness (aspects of Extraversion and Neuroticism, respectively) showed the highest $r_{v} \mathrm{~s}$ and thus higher nomological trait-state homomorphy.

Notably, Humility showed markedly different $r_{v}$ s as a function of correlates used. For aggregated daily DIAMONDS, $r_{v}$ was .82 , while it was -.32 for CAQ items. This may highlight how the careful selection of nomological correlates is important but also that a vector correlation across only eight coefficients may be less reliable than one across 100 coefficients.

\section{Nomological Lens Models}

Domains. Domain-level regressions predicting traits and state aggregates from nomological correlates (DIAMONDS, CAQ) can be found in Online Supplemental Table D for both studies. Notably, Online Supplemental Table D contains regression coefficients that control for the shared overlap between predictors (as opposed to Online Supplemental Table B). Across all domains, studies, and nomological correlates, grand averages were $G=.60$ (range: .00 for Study 2 Honesty/Humility using CAQ items to .95 for Study 2 Extraversion using aggregated daily situations) and $C=.16$ (range: -.04 for Study 2 Honesty/Humility using CAQ items to .31 for Study 2 Extraversion 
Table 2. Descriptive Statistics and Reliabilities for Variables in Studies I and 2.

\begin{tabular}{|c|c|c|c|c|c|c|}
\hline Variable & \multicolumn{3}{|c|}{ Study I } & \multicolumn{3}{|c|}{ Study 2} \\
\hline Honesty/Humility & 3.33 & 0.55 & .63 & 3.50 & 0.33 & .77 \\
\hline Honesty & - & - & - & 4.08 & 0.72 & .60 \\
\hline Humility & - & - & - & 3.88 & 0.72 & .76 \\
\hline Withdrawal & - & - & - & 3.12 & 0.75 & .80 \\
\hline eXtraversion (Extraversion) & 3.57 & 0.62 & .79 & 4.14 & 0.59 & .86 \\
\hline Enthusiasm & - & - & - & 4.29 & 0.69 & .82 \\
\hline Assertiveness & - & - & - & 3.98 & 0.68 & .80 \\
\hline Agreeableness & 3.31 & 0.63 & .75 & 4.52 & 0.49 & .80 \\
\hline Compassion & - & - & - & 4.59 & 0.60 & .81 \\
\hline Openness & 3.20 & 0.66 & .74 & 4.22 & 0.50 & .78 \\
\hline Openness & - & - & - & 4.14 & 0.64 & .78 \\
\hline Intellect & - & - & - & 4.30 & 0.60 & .68 \\
\hline \multicolumn{7}{|l|}{ Aggregated States } \\
\hline Honesty/Humility & 5.67 & 1.06 & .46 & 5.30 & 1.00 & .57 \\
\hline Honesty & - & - & - & 5.36 & 0.98 & .49 \\
\hline Humility & - & - & - & 5.23 & 1.08 & .56 \\
\hline Emotionality (Neuroticism) & 3.50 & 1.15 & .32 & 2.13 & 0.85 & .35 \\
\hline Volatility & - & - & - & 2.05 & 0.83 & .29 \\
\hline Withdrawal & - & - & - & 2.20 & 0.94 & .35 \\
\hline eXtraversion (Extraversion) & 4.70 & 1.13 & .29 & 4.80 & 0.92 & .42 \\
\hline Enthusiasm & - & - & - & 5.00 & 1.11 & .31 \\
\hline Openness & 5.12 & 1.04 & .38 & 4.71 & 0.94 & .48 \\
\hline Openness & - & - & - & 4.82 & 0.96 & .39 \\
\hline Intellect & - & - & - & 4.60 & 1.02 & .47 \\
\hline \multicolumn{7}{|l|}{ Life situations } \\
\hline Duty & 5.62 & 0.81 & .56 & - & - & - \\
\hline Intellect & 5.09 & 0.90 & .63 & - & - & - \\
\hline Adversity & 2.82 & 1.08 & .70 & - & - & - \\
\hline Mating & 3.51 & 1.08 & .60 & - & - & - \\
\hline pOsitivity & 5.21 & 0.82 & .64 & - & - & - \\
\hline Negativity & 4.30 & $\mathrm{I} .24$ & .84 & - & - & - \\
\hline Deception & 3.07 & 1.07 & .66 & - & - & - \\
\hline Sociality & 5.21 & 0.84 & .46 & - & - & - \\
\hline \multicolumn{7}{|l|}{ Aggregated daily situations } \\
\hline Duty & 4.19 & 1.16 & .23 & 3.98 & 1.26 & .23 \\
\hline Intellect & 3.35 & 1.08 & .21 & 3.15 & 1.09 & .21 \\
\hline Adversity & 1.69 & 0.84 & .36 & 1.44 & 0.75 & .34 \\
\hline Mating & 2.53 & 1.21 & .29 & 1.95 & 1.32 & .44 \\
\hline pOsitivity & 4.44 & 1.01 & .22 & 4.63 & 1.12 & .25 \\
\hline Negativity & 2.46 & 1.02 & .28 & 2.06 & 0.97 & .27 \\
\hline Deception & 1.68 & 0.82 & .36 & 1.32 & 0.75 & .52 \\
\hline Sociality & 4.04 & 1.04 & .17 & 4.33 & 1.27 & .21 \\
\hline
\end{tabular}


Table 2. (continued)

\begin{tabular}{|c|c|c|c|c|c|c|}
\hline Variable & \multicolumn{3}{|c|}{ Study I } & \multicolumn{3}{|c|}{ Study 2} \\
\hline CAQ0 I-Critical, skeptical, not easily impressed & 4.63 & 2.14 & - & 5.20 & 2.37 & - \\
\hline CAQ02-Genuinely dependable person & 7.81 & 1.23 & - & 7.90 & 1.33 & - \\
\hline CAQ03-Has a wide range of interests & 7.57 & 1.65 & - & 7.73 & 1.61 & - \\
\hline CAQ06-Is fastidious & 6.16 & 2.16 & - & 5.96 & 2.26 & - \\
\hline CAQ07-Favors conservative values & 5.15 & 2.40 & - & 4.70 & 2.63 & - \\
\hline CAQ08-High degree of intellectual capacity & 6.89 & 1.43 & - & 7.28 & 1.39 & - \\
\hline CAQ09_Uncomfortable w/uncertainty and complexities & 5.20 & 2.42 & - & 5.05 & 2.52 & - \\
\hline CAQI0-Anxiety/Tension find outlet in bodily symptoms & 4.46 & 2.61 & - & 5.04 & 2.62 & - \\
\hline CAQII-Protective of those close to him or her & 8.10 & 1.34 & - & 8.20 & 1.27 & - \\
\hline CAQ16-Introspective & 4.87 & 2.31 & - & 5.79 & 2.48 & - \\
\hline CAQI7-Sympathetic/considerate & 7.72 & 1.37 & - & 7.85 & 1.34 & - \\
\hline CAQI8_-Initiates humor & 7.56 & 1.47 & - & 7.52 & 1.61 & - \\
\hline CAQ 19-Seeks reassurance from others & 5.67 & 2.47 & - & 5.79 & 2.28 & - \\
\hline CAQ20_-Rapid personal tempo & 6.04 & 2.18 & - & 5.95 & 2.18 & - \\
\hline CAQ21-Arouses nurturant feelings in others & 5.75 & 2.04 & - & 6.30 & 1.99 & - \\
\hline CAQ22-Feels lack of personal meaning in life & 3.14 & 2.46 & - & 3.42 & 2.50 & - \\
\hline CAQ23-Transfers/projects blame & 2.85 & 2.06 & - & 3.34 & 2.07 & - \\
\hline CAQ24-Prides self on being objective/rational & 5.55 & 2.26 & - & 6.57 & 1.98 & - \\
\hline CAQ25-Delays gratification unnecessarily & 4.60 & 2.26 & - & 5.01 & 2.33 & - \\
\hline CAQ26-Productive; gets things done & 7.14 & 1.72 & - & 7.16 & 1.69 & - \\
\hline CAQ27-Condescending to others & 4.11 & 2.43 & - & 3.94 & 2.30 & - \\
\hline CAQ35-Warmth; capacity for close relationships & 7.99 & 1.30 & - & 7.89 & 1.43 & - \\
\hline CAQ36 - Negativistic; tends to undermine/sabotage & 2.65 & 1.84 & - & 3.59 & 2.27 & - \\
\hline CAQ37-Guileful, deceitful, and manipulative & 2.64 & 2.13 & - & 3.31 & 2.34 & - \\
\hline CAQ38-Hostile toward others & 2.39 & 1.76 & - & 3.00 & 2.01 & - \\
\hline CAQ39-Unconventional thought process & 5.03 & 2.46 & - & 6.40 & 2.17 & - \\
\hline CAQ40 - Generally fearful & 2.80 & 2.05 & - & 3.81 & 2.29 & - \\
\hline CAQ4I-Moralistic & 7.04 & 1.88 & - & 7.46 & 1.53 & - \\
\hline CAQ42-Reluctant to commit to course of action & 4.23 & 2.34 & - & 4.13 & 2.34 & - \\
\hline CAQ43_Facially/gesturally expressive & 6.82 & 1.90 & - & 7.28 & 1.80 & - \\
\hline CAQ44-Evaluates motivations of others & 6.74 & 1.67 & - & 7.15 & 1.53 & - \\
\hline CAQ45-Brittle ego-defense system & 3.79 & 2.26 & - & 4.15 & 2.38 & - \\
\hline CAQ46_Fantasizes/daydreams & 5.91 & 2.51 & - & 6.53 & 2.25 & - \\
\hline CAQ47-Readiness to feel guilt & 5.17 & 2.50 & - & 5.67 & 2.42 & - \\
\hline CAQ48-Keeps people at a distance & 3.31 & 2.37 & - & 3.75 & 2.38 & - \\
\hline CAQ49-Basically distrustful of others & 3.47 & 2.52 & - & 4.19 & 2.47 & - \\
\hline CAQ50-Unpredictable in behavior/attitudes & 3.68 & 2.51 & - & 4.25 & 2.50 & - \\
\hline CAQ5I_Values intellectual/cognitive matters & 7.13 & 1.54 & - & 7.84 & 1.34 & - \\
\hline CAQ52-Behaves in an assertive fashion & 5.78 & 2.21 & - & 5.85 & 2.02 & - \\
\hline CAQ53-Unable to delay gratification & 4.56 & 2.14 & - & 4.71 & 2.26 & - \\
\hline CAQ54-Emphasizes being w/others & 6.02 & 2.05 & - & 6.21 & 2.09 & - \\
\hline CAQ55-Self-defeating & 4.17 & 2.36 & - & 4.13 & 2.42 & - \\
\hline CAQ56-Responds to humor & 8.17 & 1.06 & - & 8.30 & 1.05 & - \\
\hline
\end{tabular}


Table 2. (continued)

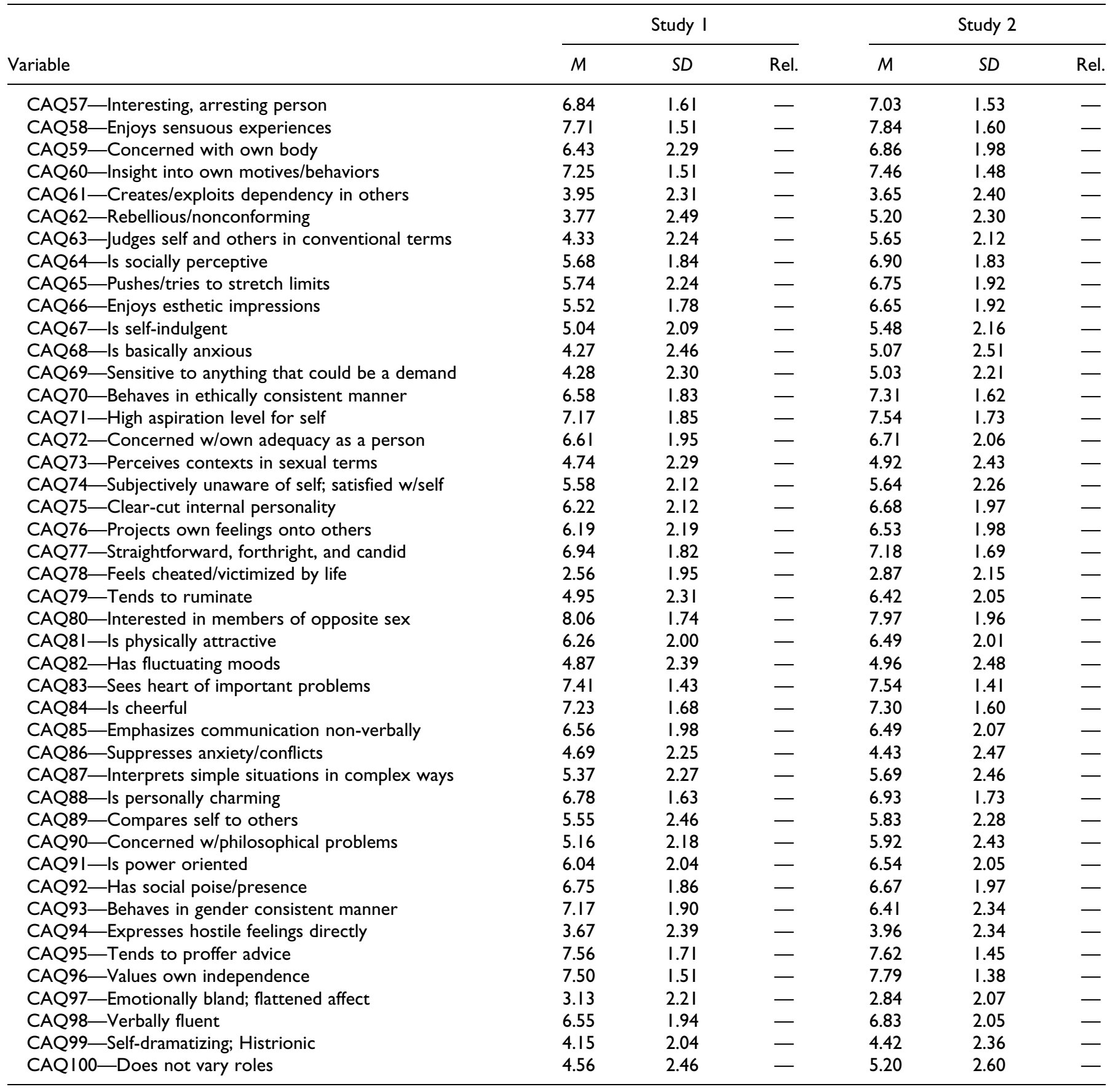

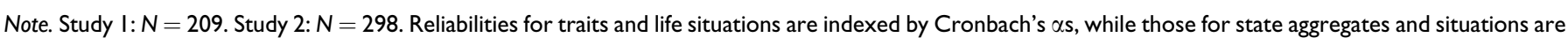

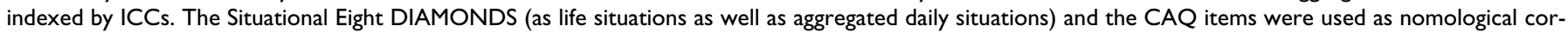

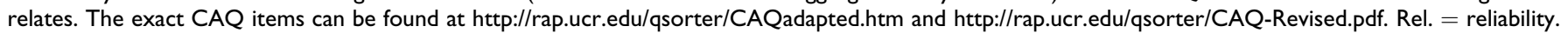

using aggregated daily situations). Typically, as summarized in the upper panel of Figure 2 under "Domains," the nomological trait-state homomorphy as indexed by $G$ was larger for eXtraversion, Conscientiousness, and Agreeableness while lower for Emotionality/Neuroticism, Honesty/Humility, and Openness.

Aspects. Aspect-level regressions predicting traits and state aggregates from nomological correlates (DIAMONDS, CAQ) can be found in Online Supplemental Table E for both studies, containing regression coefficients that control for the shared overlap between predictors (as opposed to Online Supplemental Table C). Across all aspects and nomological correlates, grand averages were $G=.55$ (range: -.15 for Humility to .88 for both Withdrawal and Enthusiasm, using aggregated daily situations) and $C=.16$ (range: .00 for Assertiveness using CAQ items to .34 for Enthusiasm using aggregated daily 
Table 3. Lens Model and Vector Correlation Statistics.

\begin{tabular}{|c|c|c|c|c|c|c|c|c|c|c|c|}
\hline Domains and Aspects & \multicolumn{7}{|c|}{ Lens Model Findings } & & & & \\
\hline \multicolumn{12}{|c|}{ Study I: Aggregated daily situations } \\
\hline Honesty/Humility & .36 & .10 & .57 & .20 & .27 & .74 & .15 & .94 & {$[.68, .99]$} & 6.52 & .001 \\
\hline Agreeableness & .33 & .05 & .67 & .25 & .29 & .83 & .16 & .99 & {$[.95,1.00]$} & 17.50 & .000 \\
\hline Conscientiousness & .29 & .05 & .46 & .25 & .33 & .76 & .27 & .89 & {$[.48, .98]$} & 4.69 & .003 \\
\hline Openness & .20 & .06 & .48 & .23 & .18 & $.1 \mathrm{I}$ & .20 & .61 & {$[-.17, .92]$} & 1.88 & .109 \\
\hline \multicolumn{12}{|l|}{ Study I: Life situations } \\
\hline Honesty/Humility & .39 & .07 & .44 & .15 & .27 & .44 & .24 & .68 & {$[-.05, .94]$} & 2.25 & .065 \\
\hline Emotionality & .48 & .10 & .17 & .06 & .18 & .53 & .16 & .67 & {$[-.06, .93]$} & 2.24 & .066 \\
\hline \multicolumn{12}{|l|}{ Study I: CAQ items } \\
\hline Honesty/Humility & .77 & .02 & .80 & .05 & .27 & .36 & .14 & .72 & {$[.6 I, .8 I]$} & 10.37 & .000 \\
\hline Emotionality & .89 & .03 & .78 & .06 & .18 & .21 & .12 & .34 & {$[.15, .50]$} & 3.55 & .001 \\
\hline eXtraversion & .92 & .02 & .78 & .06 & .37 & .50 & .06 & .90 & {$[.86, .93]$} & 20.52 & .000 \\
\hline Agreeableness & .84 & .03 & .81 & .05 & .29 & .42 & .02 & .80 & {$[.71, .86]$} & 13.08 & .000 \\
\hline Conscientiousness & .88 & .02 & .81 & .05 & .33 & .45 & .05 & .84 & {$[.77, .89]$} & 15.27 & .000 \\
\hline Openness & .84 & .03 & .80 & .06 & .18 & .21 & .14 & .53 & {$[.38, .66]$} & 6.26 & .000 \\
\hline \multicolumn{12}{|c|}{ Study 2: Aggregated daily situations } \\
\hline Honesty/Humility & .09 & .01 & .42 & .08 & -.03 & .12 & -.03 & -.05 & {$[-.73, .68]$} & -0.12 & .910 \\
\hline Honesty & .22 & .06 & 45 & .09 & .12 & .24 & .1 I & .58 & {$[-.21, .91]$} & 1.75 & .131 \\
\hline Humility & .15 & .03 & .36 & .09 & .03 & -.15 & .05 & .82 & {$[.26, .97]$} & 3.45 & .014 \\
\hline Neuroticism & .33 & .08 & .57 & .12 & .40 & .89 & .30 & .95 & {$[.73, .99]$} & 7.26 & .000 \\
\hline Conscientiousness & .30 & .06 & .39 & .11 & .36 & .85 & .30 & .93 & {$[.67, .99]$} & 6.35 & .001 \\
\hline Industriousness & .34 & .08 & .39 & .12 & .33 & .78 & .26 & .87 & {$[.44, .98]$} & 4.40 & .005 \\
\hline Orderliness & .18 & .03 & .37 & .11 & .30 & .78 & .27 & .80 & {$[.23, .96]$} & 3.31 & .016 \\
\hline Openness & .17 & .03 & .43 & $.1 I$ & .22 & .68 & .19 & .76 & {$[.11, .95]$} & 2.84 & .029 \\
\hline Openness & .17 & .04 & .44 & .10 & .25 & .85 & .21 & .86 & {$[.38, .97]$} & 4.05 & .007 \\
\hline Intellect & .18 & .04 & .40 & .14 & .15 & .19 & .15 & .16 & {$[-.61, .78]$} & 0.41 & .699 \\
\hline \multicolumn{12}{|l|}{ Study 2: CAQ items } \\
\hline Honesty/Humility & .62 & .01 & .67 & .04 & -.03 & .00 & -.04 & .18 & {$[-.02, .36]$} & $|.8|$ & .073 \\
\hline Honesty & .75 & .03 & .66 & .04 & .12 & .15 & .10 & .53 & {$[.37, .66]$} & 6.17 & .000 \\
\hline Humility & .78 & .02 & .68 & .04 & .03 & -.04 & .12 & -.32 & {$[-.49,-.13]$} & -3.34 & .001 \\
\hline Neuroticism & .91 & .02 & .71 & .03 & .40 & .57 & .11 & .93 & {$[.89, .95]$} & 24.42 & .000 \\
\hline Volatility & .86 & .02 & .69 & .03 & .30 & .46 & .07 & .87 & {$[.81, .91]$} & 17.55 & .000 \\
\hline Withdrawal & .90 & .02 & .72 & .03 & .43 & .62 & .10 & .94 & {$[.92, .96]$} & 27.92 & .000 \\
\hline Extraversion & .90 & .01 & .72 & .04 & .41 & .62 & .03 & .95 & {$[.92, .96]$} & 29.24 & .000 \\
\hline Enthusiasm & .88 & .02 & .72 & .04 & .42 & .62 & .09 & .92 & {$[.89, .95]$} & 23.77 & .000 \\
\hline Assertiveness & .86 & .02 & .71 & .04 & .32 & .51 & .00 & .91 & {$[.87, .94]$} & 21.66 & .000 \\
\hline Agreeableness & .85 & .02 & .67 & .04 & .27 & .37 & .14 & .73 & {$[.62, .81]$} & 10.58 & .000 \\
\hline Compassion & .82 & .02 & .68 & .04 & .28 & .44 & .09 & .79 & {$[.70, .85]$} & $|2.8|$ & .000 \\
\hline Politeness & .80 & .02 & .66 & .04 & .19 & .20 & .17 & .56 & {$[.41 ; .68]$} & 6.75 & .000 \\
\hline Conscientiousness & .86 & .02 & .70 & .03 & .36 & .49 & .18 & .91 & {$[.87, .94]$} & 22.16 & .000 \\
\hline Industriousness & .88 & .02 & .68 & .03 & .33 & .52 & .04 & .92 & {$[.89, .95]$} & 23.45 & .000 \\
\hline Orderliness & .80 & .02 & .70 & .04 & .30 & .36 & .23 & .79 & {$[.70, .85]$} & 12.78 & .000 \\
\hline
\end{tabular}


Table 3. (continued)

\begin{tabular}{|c|c|c|c|c|c|c|c|c|c|c|c|}
\hline Domains and Aspects & \multicolumn{7}{|c|}{ Lens Model Findings } & & & & \\
\hline Openness & .83 & .02 & .68 & .04 & .22 & .36 & .03 & .66 & {$[.54, .76]$} & 8.80 & .000 \\
\hline Openness & .84 & .02 & .65 & .03 & .25 & .42 & .06 & .79 & {$[.71, .86]$} & 12.86 & .000 \\
\hline
\end{tabular}

Note. Study I: $N=209$. Study 2: $N=298$. The Situational Eight DIAMONDS $(k=8)$ and the California Adult Q-Sort $(C A Q)$ items $(k=100)$ were treated as nomological correlates. Bold-faced indices and values are "preferred" ones: DIAMONDS with eight predictors is preferred over CAQ with 100 items for the nomological lens model approach; and CAQ with 100 correlates is preferred over DIAMONDS with 8 predictors for the nomological vector-correlation approach. Rtrait = trait saturation (multiple correlation); Abs. b̆trait = average of absolute regression coefficients for trait saturations in Table B (left side); Rstate = state saturation (multiple correlation); Abs. b̄state = average of absolute regression coefficients for aggregated state saturations in Table B (right side); $r=$ regular correlation between a trait and a corresponding aggregated state (trait-state manifestation, achievement); $G=$ nomological similarity or matching index; $C=$ residual correlation index; $r v=$ vector (profile) correlation (across eight correlates: $d f=6$; across 100 correlates: $d f=98$ ), with those not statistically significant at $p<.05$ appearing in light gray.

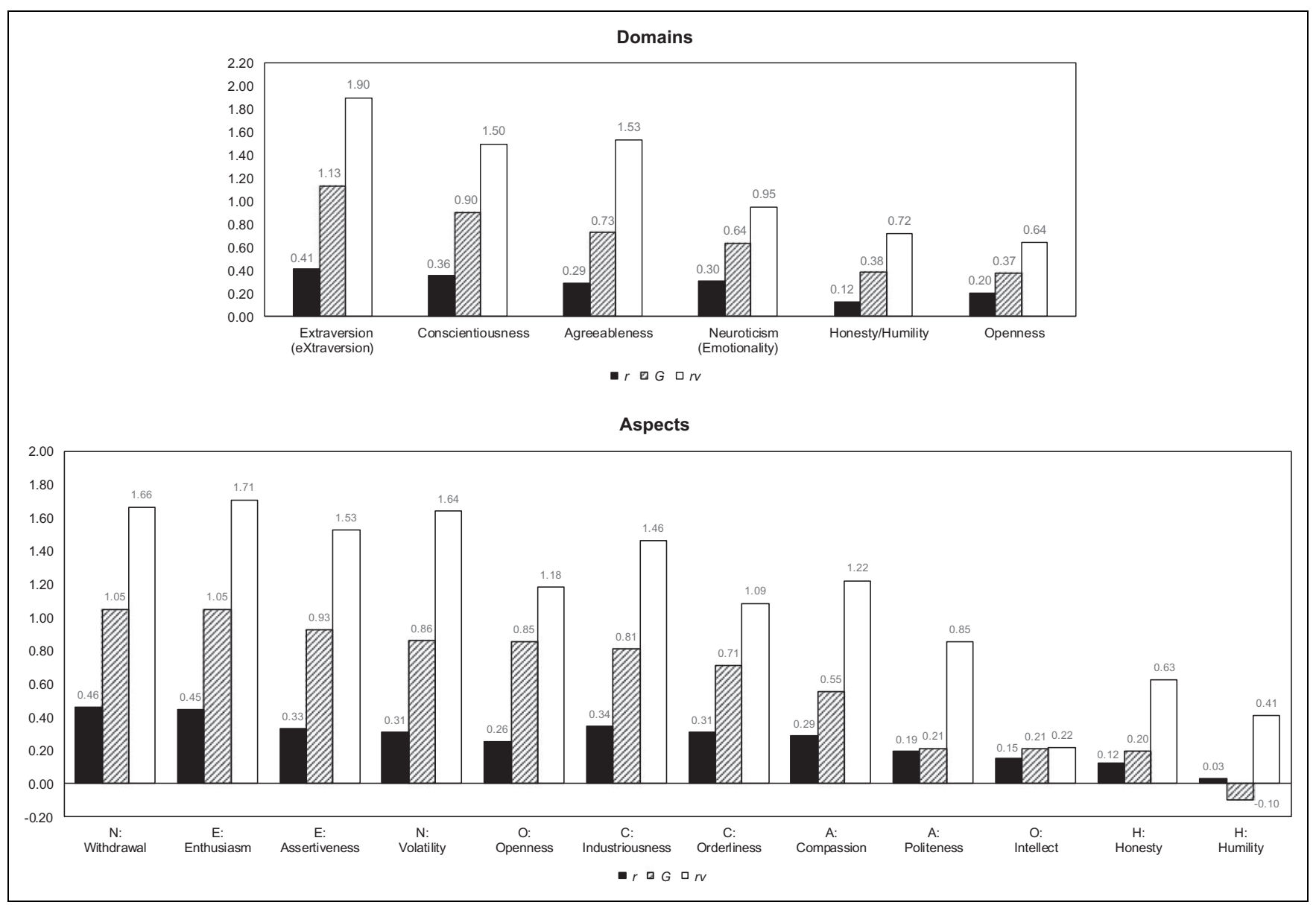

Figure 2. Averaged trait-state homomorphy indices per domain and aspects. $y$-axis $=r$-to-z transformed correlation coefficients. $x$-axis: Domains or aspects sorted according to descending $G$. Domains or aspects on the left show more nomological trait-state homomorphy, domains, or aspects on the right less. Domain and aspect scores from Table 3 were used. Domain labels of aspects (lower panel): $\mathrm{H}=\mathrm{Honesty/}$ Humility, $\mathrm{N}=$ Neuroticism, $\mathrm{E}=$ Extraversion, $\mathrm{A}=$ Agreeableness, $\mathrm{C}=$ Conscientiousness, $\mathrm{O}=\mathrm{Openness.} \mathrm{Average} \mathrm{findings} \mathrm{per} \mathrm{domain} \mathrm{were}$ computed across Studies I and 2 and those per aspect only in Study 2. $r$ (black bars) = zero-order correlation between a trait and an aggregated state (trait-state manifestation), $G$ (striped bars) = nomological similarity index, $r_{v}$ (white bars) $=$ vector correlations. As all coefficients are essentially correlations, they are depicted in $r$-to-z transformed units. 
Table 4. Replication of Rank-Orderings Among Domains and Aspects.

\begin{tabular}{|c|c|c|c|c|c|c|c|c|c|c|c|c|c|c|}
\hline \multirow[b]{3}{*}{ Scales } & \multicolumn{2}{|c|}{$\begin{array}{l}\text { Trait-State } \\
\text { Manifestations } r\end{array}$} & \multicolumn{4}{|c|}{$\begin{array}{l}\text { Eight Aggregated Daily } \\
\text { Situation Characteristics }\end{array}$} & \multicolumn{4}{|c|}{$\begin{array}{l}\text { Eight Characteristics } \\
\text { of Life Situations }\end{array}$} & \multicolumn{4}{|c|}{100 CAQ Person Descriptors } \\
\hline & \multirow[b]{2}{*}{ Study I } & \multirow[b]{2}{*}{ Study 2} & \multicolumn{2}{|c|}{ Study I } & \multicolumn{2}{|c|}{ Study 2} & \multicolumn{2}{|c|}{ Study I } & \multicolumn{2}{|c|}{ Study 2} & \multicolumn{2}{|c|}{ Study I } & \multicolumn{2}{|c|}{ Study 2} \\
\hline & & & $G$ & $r_{v}$ & $G$ & $r_{v}$ & $G$ & $r_{v}$ & $G$ & $r_{v}$ & $G$ & $r_{v}$ & $G$ & $r_{v}$ \\
\hline \multicolumn{15}{|l|}{ Domains } \\
\hline Honesty/Humility & 4 & 6 & 4 & 2 & 6 & 6 & 5 & 4 & - & - & 4 & 4 & 6 & 6 \\
\hline Emotionality/Neuroticism ${ }^{\mathrm{a}}$ & 5 & 2 & 5 & 6 & 2 & 2 & 4 & 5 & - & - & 5 & 6 & 2 & 2 \\
\hline eXtraversion/Extraversion ${ }^{\mathrm{b}}$ & I & I & 2 & 3 & I & I & I & 1 & - & - & 1 & I & I & I \\
\hline Agreeableness & 3 & 4 & I & I & 5 & 4 & 3 & 3 & - & - & 3 & 3 & 4 & 4 \\
\hline Conscientiousness & 2 & 3 & 3 & 4 & 3 & 3 & 2 & 2 & - & - & 2 & 2 & 3 & 3 \\
\hline Openness & 6 & 5 & 6 & 5 & 4 & 5 & 6 & 6 & - & - & 6 & 5 & 5 & 5 \\
\hline \multicolumn{15}{|l|}{ Aspects $^{c}$} \\
\hline \multicolumn{15}{|l|}{ Honesty/Humility } \\
\hline Honesty & - & 10 & - & - & 7 & 11 & - & - & - & - & - & - & 10 & 7 \\
\hline Humility & - & II & - & - & 10 & 8 & - & - & - & - & - & - & 11 & 9 \\
\hline \multicolumn{15}{|l|}{ Neuroticism } \\
\hline Volatility & - & 5 & - & - & 4 & I & - & - & - & - & - & - & 4 & 4 \\
\hline Withdrawal & - & 1 & - & - & I & 3 & - & - & - & - & - & - & 1 & I \\
\hline \multicolumn{15}{|l|}{ Extraversion } \\
\hline Enthusiasm & - & 2 & - & - & I & 2 & - & - & - & - & - & - & 1 & 2 \\
\hline Assertiveness & - & 4 & - & - & 2 & 4 & - & - & - & - & - & - & 3 & 3 \\
\hline \multicolumn{15}{|l|}{ Agreeableness } \\
\hline Compassion & - & 6 & - & - & 6 & 5 & - & - & - & - & - & - & 5 & 5 \\
\hline Politeness & - & 8 & - & - & 8 & 10 & - & - & - & - & - & - & 9 & 6 \\
\hline \multicolumn{15}{|l|}{ Conscientiousness } \\
\hline Industriousness & - & 3 & - & - & 5 & 6 & - & - & - & - & - & - & 2 & 2 \\
\hline Orderliness & - & 5 & - & - & 5 & 9 & - & - & - & - & - & - & 7 & 5 \\
\hline \multicolumn{15}{|l|}{ Openness } \\
\hline Openness & - & 7 & - & - & 3 & 7 & - & - & - & - & - & - & 6 & 5 \\
\hline Intellect & - & 9 & - & - & 9 & 12 & - & - & - & - & - & - & 8 & 8 \\
\hline
\end{tabular}

Note. Rank-orders are given such that smaller numbers indicate more trait-state homomorphy (e.g., I is the highest possible value) and larger numbers decreasingly lower homomorphy. Ties got the same rank. Ties were possible if correlations were identical when rounded to two decimals. Bold-faced ranks mean that the respective index $\left(G\right.$ or $\left.r_{v}\right)$ may be favored in that instance. The nomological vector-correlation approach, with the index $r_{v}$, should be favored when using many correlations (I00 CAQ items) as opposed to few (Eight DIAMONDS). The Nomological Lens Model Approach, with the index $G$, should be favored when using few predictors (Eight DIAMONDS) as opposed to many (I00 CAQ items).

${ }^{a}$ Emotionality (within the HEXACO model) was used for Study I, and Neuroticism (within the Big Five Aspects model) in Study 2. beXtraversion (within the HEXACO model) was used for Study I, and Extraversion (within the Big Five Aspects model) in Study 2. ${ }^{\mathrm{C}}$ The aspects showed multiple rank ties. Thus, the last possible ranks ranged from 9 to 12 .

situations). Typically, as summarized in the lower panel of Figure 2 under "Aspects," the nomological trait-state homomorphy as indexed by $G$ was larger for Withdrawal, Enthusiasm, Assertiveness, and Volatility (aspects of Extraversion and Neuroticism, respectively) while lower for Intellect, Honesty, and Humility.

\section{Replication}

To gauge levels of replicability across both studies and/or across sets of nomological correlates (life situations vs. aggregated daily situations vs. CAQ), Table 4 summarizes findings from Table 3 in terms of rank ordering domains and aspects. While there was no perfect replication, the general pattern of findings replicated fairly well with only few exceptions.

\section{Discussion}

\section{Summary and Interpretation}

Using a nomological perspective, we proposed a way of estimating the extent to which traits and state aggregates actually tap the same construct-in addition to looking at the raw convergent correlation (trait-state manifestation $r$ ). If a trait and a corresponding state aggregate show the same associations with a set of correlates, then both likely tap the same construct as defined per a common nomological network. Across both studies, convergent correlations indexing trait-state manifestations in daily life were rather modest, while the typical nomological trait-state homomorphy was quite sizable although differed substantially by domain and aspect (Figure 2). Specifically, trait and state aggregate assessments of eXtraversion, Agreeableness, and Conscientiousness were particularly nomologically homomorphous, while those for Honesty/Humility, 
emotionality, and Openness were less so. This general picture notwithstanding, there are some specific observations to make when looking at Table 4.

First, the central indices $r_{v}$ for the NoVeCA and $G$ for the NoLeMA did not show perfect convergence but still converged quite often when rank ordering domains and aspects. Thus, more often than not, we would reach the same conclusions. However, a NoVeCA will be strongest for many and a NoLeMA for few correlates. We opted to show findings for both approaches to facilitate vis-à-vis comparisons, but NoVeCA findings with the CAQ items as correlates and NoLeMA findings with the DIAMONDS as predictors may be deemed more appropriate, respectively (see bold-faced values in Tables 3 and 4).

Second, as evident from Table 4 and Figure 2, eXtraversion/Extraversion, in terms of a domain and its aspects (especially Enthusiasm), was the most nomologically homomorphous. In contrast, Openness (especially the Intellect aspect) and Honesty/Humility uniformly showed low nomological homomorphy - in other words, state aggregates were picking up something else than trait scales. The matter was more complex for emotionality/Neuroticism and Agreeableness. Emotionality within the HEXACO model (Study 1) was less and Neuroticism within the Big Five Aspects model (Study 2) more nomologically homomorphous; this was reversed for Agreeableness which was more homomorphous in Study 1 than in Study 2 (see Table 4). These findings alert us to the pragmatic truth that constructs are operationalized in both conceptual and measurement terms. In our case, not only did the measures differ between Studies 1 and 2 but also their conceptual foundations. Specifically, HEXACO emotionality and Agreeableness represent rotated variants of more "traditional" Big Five scales (e.g., Ashton \& Lee, 2007, p. 152). Thus, HEXACO Emotionality and Agreeableness scales may behave differently than BFAS scales.

As we formed no a priori hypotheses which domains or aspects would show more or less nomological trait-state homomorphy, we can only speculate on the observed differences in Table 4 and Figure 2. However, it seems that, for some reason, rather observable and social dimensions (e.g., being extraverted, enthusiastic, assertive, withdrawn, volatile, and conscientious) tended toward more while those less observable, requiring more introspection, and pertaining more to internal mental processes (e.g., being anxious, intellectual, honest, and humble) tended toward less homomorphy. Interestingly, these findings stand in line with other research demonstrating that less observable and behaviorally manifest traits, such as Neuroticism and Openness, coincide with lesser self-other agreement (Funder \& Dobroth, 1987; John \& Robins, 1993; Vazire, 2010) and less true trait variance in single other ratings (Rauthmann, 2017).

\section{Future Directions, Recommendations, and Caveats}

The raw correlation between a trait and a state aggregate (traitstate manifestation) is already important and meaningful information. However, in psychometric terms, this is only evidence for convergent validity. We suggest that researchers additionally make use of nomological validity by attending to the similarity of nomological networks of trait and state aggregate measures. We anticipate that our nomological perspective would be especially useful when trait- and state measures are not entirely commensurate, for example, when (a) different items (in nature and number) were used, (b) response scale units are not the same, or (c) the constructs are similar yet still different (e.g., the trait is measured as a domain vs. the state as an aspect, facet, or nuance; DeYoung et al., 2007; Mõttus, Kandler, Bleidorn, Riemann, \& McCrae, 2017). More generally, however, our approach can help empirically address jingle (different constructs/scales are erroneously presumed to be identical because they share the same label) and jangle problems (identical constructs/scales are erroneously presumed to be different because they each have a different label).

Our findings harbor implications for (short) scale development: More care could be devoted to constructing nomologically homomorphous trait- and state measures for certain domains or aspects. Researchers may, for example, examine the "ABCD" (affect, behavior, cognition, and desire) content of items and scales (Wilt \& Revelle, 2015). Those scales with more affective, cognitive, and/or motivational or less behavioral content could show less trait-state homomorphy although this remains a hypothesis to be tested specifically in further research.

The NoVeCA and NoLeMA presented here are flexible and can be extended to other person characteristics (e.g., selfesteem and happiness) that may be assessed as traits and states. Moreover, because nomological networks are defined by the sample of correlates used, the networks of focal constructs need to be selected with great care. Theory or previous empirical work may guide which correlates to select and how they are associated with the focal scales. In some cases, there may be no prior theory to rely on, or existing theories provide no clear recommendations for nomological networks. Then, prior empirical evidence is more informative, but this could entail a too narrow sampling of correlates. While a representative set of correlates would be desirable, key questions are which variables such a "representative" set would encompass and whether measurement tools are available. As trait-state homomorphy is quantified in relation to a common set of nomological correlates, findings may change somewhat depending on which correlates are chosen (see Table 4). Ideally, a chosen set would balance correlates (of similar or identical reliability) that should be positively, negatively, and barely associated with the focal construct. Thus, we recommend that future research replicates and extends our findings (specifically the rank orderings in Table 4 and Figure 2) using different nomological correlates, trait- and state measures, and samples (e.g., community samples and other countries).

We advise researchers to carefully select a NoVeCA and NoLeMA. The NoVeCA works best with many correlates, but the theoretical relevance, reliability, intercorrelations, and labeling of the correlates need to be considered. Further, the 
NoVeCA may be improved by using modified correlational indices ( $r_{\text {alerting- } C V}$ and $r_{\text {contrast- } C V}$ ) proposed in the quantification of construct validity approach by Westen and Rosenthal (2003), although their interpretation and computation has so far not been easy. The NoLeMA works best with few correlates, but the same issues as in the NoVeCA need to be considered plus the fact that each predictor is being controlled for all other ones, and such partialling may create issues of interpretability (What is the single predictor residually capturing when everything else is being held constant?).

We have left open what constitutes a high (or low) $G$ or $r_{v}$. This question may only be explored once more data come in and empirically derived cutoffs can be defined. For now, we endorse comparing domains, aspects, or facets relatively to each other (see Figure 2). However, perfect comparability is only given with identical amounts of items and reliabilities of trait and state aggregate scales and when the same sets of correlates were used.

It is also important to think about conceptual and methodological forms of asymmetries between traits and states. Conceptually, traits are something different than states (Baumert et al., 2017). Although the complete observation of all states (within a long enough period) should approximate a trait reasonably well, short "bursts" of measurement (such as in experience sampling) may not be sufficient to fully get at the trait. Methodologically, traits and states are often operationalized via self-reports (as done here) and not via actual behavior, although states are actually tied more closely to concrete behavior. Further, traits are usually measured with several items, while states in experience sampling often with single or very few items. Thus, there are asymmetries between trait- and state measures in breadth and reliability. These issues can impact findings in Table 3.

\section{Conclusion}

Convergent correlations between self-reported traits and aggregated states from repeated measurements are not sufficient alone to establish trait-state homomorphy (i.e., whether the same construct is being measured). While high trait-state manifestation correlations are desirable, it is more stringent to test to what extent the nomological networks of traits and state aggregates are similar to each other. Within a nomological perspective manifesting in a NoVeCA and NoLeMA, we introduced the concept of nomological trait-state homomorphy: Measures of a trait and state aggregate capture the same construct when both show relatively similar nomological associations to a common set of correlates. We found that trait and state aggregate measures of some domains (e.g., eXtraversion/Extraversion) showed stronger nomological homomorphy than others (e.g., Openness and Honesty/Humility). Although we framed our nomological perspective in terms of the question to what extent traits and state aggregates capture the same constructs, it is more general and can be used whenever two scales should be examined for homomorphy. Our approaches can thus be flexibly applied to other traits, states, and correlates.

\section{Declaration of Conflicting Interests}

The author(s) declared no potential conflicts of interest with respect to the research, authorship, and/or publication of this article.

\section{Funding}

The author(s) disclosed receipt of the following financial support for the research, authorship, and/or publication of this article: Study 2 was supported by NSF grant \#1420105 to Ryne Sherman.

\section{Supplemental Material}

The supplemental material is available in the online version of the article.

\section{Notes}

1. Sometimes stability of the nomological network across time points also needs to be examined because measurements of scales are far apart (e.g., childhood-adulthood). This is not the case in experience sampling studies where trait, state, and correlate reports are usually provided in proximity to each other (Fleeson \& Gallagher, 2009).

2. In the case of too many single correlates and/or them being too highly intercorrelated, it is possible to run a principal component analysis over all correlates and then use the resulting dimensions as correlates. If the correlates cannot or should not be reduced, then the nomological vector correlation approach is advisable.

3. It may vice versa also concern how a state aggregate approximates a trait.

\section{References}

Asendorpf, J. B., \& van Aken, M. A. G. (2003). Validity of big five personality judgments in childhood: A 9-year longitudinal study. European Journal of Personality, 17, 1-17.

Ashton, M. C., \& Lee, K. (2007). Empirical, theoretical, and practical advantages of the HEXACO model of personality structure. Personality and Social Psychology Review, 11, 150-166.

Ashton, M. C., \& Lee, K. (2009). The HEXACO-60: A short measure of the major dimensions of personality. Journal of Personality Assessment, 91, 340-345.

Augustine, A. A., \& Larsen, R. J. (2012). Is a trait really the mean of states? Journal of Individual Differences, 33, 131-137.

Bates, D., Maechler, M., Bolker, B., \& Walker, S. (2014). Ime4: Linear mixed-effects models using Eigen and S4. http://cran.r-project. org/package $=1 m e 4$. Retrieved fromhttp://cran.r-project.org/ package $=\operatorname{lme} 4$

Baumert, A., Schmitt, M., Perugini, M., Johnson, W., Blum, G., Borkenau, P., . . Jayawickreme, E. (2017). Integrating personality structure, personality process, and personality development. European Journal of Personality, 31(5), 503-528.

Bem, D. J., \& Funder, D. C. (1978). Predicting more of the people more of the time: Assessing the personality of situations. Psychological Review, 85, 485-501.

Block, J. (1961), The Q-sort method in personality assessment and psychiatric research. Springfield, IL: Charles C. Thomas. 
Block, J. (2008). The Q-sort in character appraisal: Encoding subjective impressions of persons quantitatively. Washington, DC: American Psychological Association.

Brown, N. A., Blake, A. B., \& Sherman, R. A. (2017). A snapshot of the life as lived: Wearable cameras in social and personality psychological science. Social Psychological and Personality Science, 8, 592-600.

Brunswik, E. (1934). Wahrnehmung und Gegenstandswelt. Grundlegung einer psychologie vom Gegenstand her [In German]. Leipzig und Wien: F. Deuticke, Australien.

Brunswik, E. (1943). Organismic achievement and environmental probability. Psychological Review, 50, 255-272.

Brunswik, E. (1952). The conceptual framework of psychology. (International Encyclopedia of Unified Science, Volume 1, Number 10.) Chicago, IL: The University of Chicago Press.

Campbell, D. T., \& Fiske, D. W. (1959). Convergent and discriminant validation by the multitrait-multimethod matrix. Psychological Bulletin, 56, 81-105.

Castellan, N. J., Jr. (1992). Relations between linear models: Implications for the lens model. Organizational Behavior and Human Decision Processes, 51, 364-381.

Cronbach, L. J., \& Meehl, P. E. (1955). Construct validity in psychological tests. Psychological Bulletin, 52, 281-302.

Dawes, R. M., \& Corrigan, B. (1974). Linear models in decision making. Psychological Bulletin, 81, 95-106.

Denissen, J. J. A., Geenen, R., Selfhout, M. H. W., \& van Aken, M. A. G. (2008). Single-item big five ratings in a social network design. European Journal of Personality, 22, 37-54.

DeYoung, C. G., Quilty, L. C., \& Peterson, J. (2007). Between facets and domains: 10 aspects of the big five. Journal of Personality and Social Psychology, 93, 880-896.

Dragulescu, A. A. (2014). xlsx: Read, write, format Excel 2007 and Excel 97/2000/XP/2003 files. Retrieved from https://cran.r-proj ect.org $/$ package $=x l s x$

Einhorn, H. J., \& Hogarth, R. M. (1975). Unit weighting schemes for decision making. Organizational Behavior and Human Performance, 13, 171-192.

Finnigan, K. M., \& Vazire, S. (2017). The incremental validity of average state self-reports over global self-reports of personality. Journal of Personality and Social Psychology. http://dx.doi.org/ $10.1037 / \mathrm{pspp} 0000136$

Fleeson, W. (2001). Toward a structure- and process-integrated view of personality: Traits as density distributions of states. Journal of Personality and Social Psychology, 80, 1011-1027.

Fleeson, W. (2007). Situation-based contingencies underlying traitcontent manifestation in behavior. Journal of Personality, 75, 825-862.

Fleeson, W. (2012). Perspectives on the person: Rapid growth and opportunities for integration. In K. Deaux \& M. Snyder (Eds.), The Oxford Handbook of Personality and Social Psychology (pp. 33-63). New York, NY: Oxford University Press.

Fleeson, W., \& Gallagher, P. (2009). The implications of big five standing for the distribution of trait manifestation in behavior: Fifteen experience-sampling studies and a meta-analysis. Journal of Personality and Social Psychology, 97, 1097-1114.

Fleeson, W., \& Jayawickreme, E. (2015). Whole trait theory. Journal of Research in Personality, 56, 82-92.
Funder, D. C. (2001). Personality. Annual Review of Psychology, 52, 197-221.

Funder, D. C. (2006). Towards a resolution of the personality triad: Persons, situations and behaviors. Journal of Research in Personality, 40, 21-34.

Funder, D. C. (2008). Persons, situations and person-situation interactions. In O. P. John, R. Robins \& L. Pervin (Eds.), Handbook of Personality (3rd ed., pp. 568-580). New York, NY: Guilford.

Funder, D. C., \& Dobroth, K. M. (1987). Differences between traits: Properties associated with interjudge agreement. Journal of Personality and Social Psychology, 52, 409-418.

Gorman, C. D., Clover, W. H., \& Doherty, M. E. (1978). Can we learn anything about interviewing real people from "interviews" of paper people? Two studies of the external validity of a paradigm. Organizational Behavior and Human Performance, 22, 165-192.

Hammond, K. R. (1980). Introduction to Brunswikian theory and methods. New Directions for Methodology of Social and Behavioral Science, 3, 1-11.

Heller, D., Komar, J., \& Lee, W. B. (2007). The dynamics of personality states, goals, and well-being. Personality and Social Psychology Bulletin, 33, 898-910.

Hogarth, R. M., \& Karelaia, N. (2007). Heuristic and linear models of judgment: Matching rules and environments. Psychological Review, 114, 733.

Horstmann, K. T., \& Rauthmann, J. F. (in preparation). How many states make a trait? A comprehensive meta-analysis of experience-sampling studies.

Horstmann, K. T., \& Ziegler, M. (in press). Situational perception and affect: Twins reared apart? Personality and Individual Differences. doi:10.1016/j.paid.2018.01.020

Hough, L. M., Oswald, F. L., \& Ock, J. (2015). Beyond the big five: New directions for personality research and practice in organizations. Annual Review of Organizational Psychology and Organizational Behavior, 2, 183-209.

John, O. P., \& Robins, R. W. (1993). Determinants of interjudge agreement: The big five, observability, evaluativeness, and the unique perspective of the self. Journal of Personality, 61, 521-551

Jones, A. B., Brown, N. A., Serfass, D. G., \& Sherman, R. A. (2017). Personality and density distributions of behaviors, emotions, and situations. Journal of Research in Personality, 69, 225-236.

Karelaia, N., \& Hogarth, R. M. (2008). Determinants of linear judgment: A meta-analysis of lens model studies. Psychological Bulletin, 134, 404-426.

Lee, K. L., \& Ashton, M. C. (in press). Psychometric properties of the HEXACO-100. Assessment. https://doi.org/10.1177/1073191116659134

Loevinger, J. (1957). Objective tests as instruments of psychological theory. Psychological Reports, 3, 635-694.

Miller, J. D., Hyatt, C. S., Maples-Keller, J. L., Carter, N. T., \& Lynam, D. R. (2017). Psychopathy and Machiavellianism: A distinction without a difference? Journal of Personality, 85, 439-453.

Measelle, J. R., John, O. P., Ablow, J. C., Cowan, P. A., \& Cowan, C. P. (2005). Can children provide coherent, stable, and valid selfreports on the big five dimensions? A longitudinal study from ages 5 to 7. Journal of Personality and Social Psychology, 89, 90-106.

Mõttus, R., Kandler, C., Bleidorn, W., Riemann, R., \& McCrae, R. R. (2017). Personality traits below facets: The consensual validity, 
longitudinal stability, heritability, and utility of personality nuances. Journal of Personality and Social Psychology, 112, 474-490.

Nestler, S., \& Back, M. D. (2013). Applications and extensions of the lens model to understand interpersonal judgments at zero acquaintance. Current Directions in Psychological Science, 22, 374-379.

Rauthmann, J. F. (2016). Motivational factors in the perception of psychological situation characteristics. Social and Personality Psychology Compass, 10, 92-108.

Rauthmann, J. F. (2017). What are other-rated scales composed of? Sources of measurement error and true trait variance in otherratings of the big five. Journal of Research in Personality, 70, $45-55$.

Rauthmann, J. F., Gallardo-Pujol, D., Guillaume, E. M., Todd, E., Nave, C. S., Sherman, R. A., .. . Funder, D. C. (2014). The situational eight DIAMONDS: A taxonomy of major dimensions of situation characteristics. Journal of Personality and Social Psychology, 107, 677-718.

Rauthmann, J. F., Jones, A. B., \& Sherman, R. A. (2016). Directionality of person-situation transactions: Are there spill-overs among and between situation experiences and personality states? Personality and Social Psychology Bulletin, 42, 893-909.

Rauthmann, J. F., \& Sherman, R. A. (2016a). Ultra-brief measures for the situational eight DIAMONDS domains. European Journal of Psychological Assessment, 32, 165-174.

Rauthmann, J. F., \& Sherman, R. A. (2016b). Measuring the situational eight DIAMONDS characteristics of situations: An optimization of the RSQ-8 to the S8*. European Journal of Psychological Assessment, 32, 155-164.

Rauthmann, J. F., Sherman, R. A., Nave, C. S., \& Funder, D. C. (2015). Personality-driven situation experience, contact, and construal: How people's personality traits predict characteristics of their situations in daily life. Journal of Research in Personality, 55, 98-111.

R Core Team (2016). R: A language and environment for statistical computing. R Foundation for Statistical Computing, Vienna, Austria. http://www.R-project.org/

Revelle, W. (2014). psych: Procedures for Psychological, Psychometric, and Personality Research. http://CRAN.R-project.org/ package $=$ psych Version $=1.4 .5$

Roberts, B. W., \& DelVecchio, W. F. (2000). The rank-order consistency of personality traits from childhood to old age: A quantitative review of longitudinal studies. Psychological Bulletin, 126, 3-25.

Roberts, B. W., Walton, K. E., \& Viechtbauer, W. (2006). Patterns of mean-level change in personality traits across the life course: A meta-analysis of longitudinal studies. Psychological Bulletin, $132,1-25$.

Robinson, D. (2016). Broom: Convert statistical analysis objects into tidy data frames. Retrieved from https://cran.r-project.org/ package $=$ broom

RStudio Team (2015). RStudio: Integrated development environment for R. Boston, MA. Retrieved from http://www.rstudio.com/

Sherman, R. A. (2015). Multicon: Multivariate constructs. Retrieved from https://cran.r-project.org/package $=$ multicon

Sherman, R. A., Rauthmann, J. F., Brown, N. A., Serfass, D. S., \& Jones, A. B. (2015). The independent effects of personality and situations on real-time expressions of behavior and emotion. Journal of Personality and Social Psychology, 109, 872-888.
Sherman, R. A., \& Serfass, D. G. (2015). The comprehensive approach to analyzing multivariate constructs. Journal of Research in Personality, 54, 40-50.

Sherman, R. A., \& Wood, D. (2014). Estimating the expected replicability of a pattern of correlations and other measures of association. Multivariate Behavioral Research, 49, 17-40.

Tucker, L. R. (1964). A suggested alternative formulation in the developments by Hursch, Hammond, and Hursch and by Hammond, Hursch, and Todd. Psychological Review, 71, 528-530.

Vazire, S. (2010). Who knows what about a person? The SELF-Other Knowledge Asymmetry (SOKA) model. Journal of Personality and Social Psychology, 98, 281-300.

Westen, D., \& Rosenthal, R. (2003). Quantifying construct validity: Two simple measures. Journal of Personality and Social Psychology, 84, 608-618.

Wickham, H. (2016). Purrr: Functional programming tools. Retrieved from https://cran.r-project.org/package $=$ purrr

Wilt, J., \& Revelle, W. (2015). Affect, behaviour, cognition and desire in the big five: An analysis of item content and structure. European journal of personality, 29, 478-497.

Xie, Y. (2016). Knitr: A general-purpose package for dynamic report generation in R. Retrieved from https://cran.r-project.org/ package $=$ knitr

\section{Author Biographies}

John F. Rauthmann is the incoming chair of personality psychology and psychological assessment at the University of Lübeck (Germany), having previously served as an Assistant Professor at the Wake Forest University, North Carolina (USA). He received his diploma from the Leopold-Franzens University, Innsbruck, and his PhD from the Humboldt-University, Berlin. He is interested in personality and individual differences, person-situation transactions, and psychological situations.

Kai T. Horstmann is a PhD student at Humboldt-University, Berlin, in the Department of Psychological Diagnostics supervised by Dr Matthias Ziegler. He received his Master's degree from HumboldtUniversity in 2015 with a focus on methods and statistics. In his research, he focuses on the interplay of situations, affect, and behavior, the application and development of situational taxonomies, the relation of personality states and personality traits, and the identification of the morally exceptional in everyday life.

Ryne A. Sherman is the Chief Science Officer at Hogan Assessment Systems and was previously an Associate Professor at Texas Tech University and Florida Atlantic University. He received his $\mathrm{PhD}$ at the University of California, Riverside in 2011. He is interested in personality, organizational effectiveness, data analytics, psychological situations, and person-situation transactions in daily life. He was recognized as an APS Rising Star in 2015 and was a recipient of the SAGE Young Scholar Award in 2018. Dr. Sherman served as an associate editor for the Journal of Research in Personality from 2016 through 2017 and is on the editorial board at numerous journals including the Journal of Personality and Social Psychology and the Journal of Personality.

Handling Editor: Wiebke Bleidorn 